\title{
Molecular Pathogenesis of Spinocerebellar Ataxia Type 6
}

\author{
Holly B. Kordasiewicz* and Christopher M. Gomez ${ }^{\dagger}$ \\ *Ludwig Institute for Cancer Research, University of California at San Diego, La Jolla, California 92093; ${ }^{\dagger}$ Department of \\ Neurology, University of Chicago, Chicago, Illinois 60637
}

\begin{abstract}
Summary: Spinocerebellar ataxia type 6 (SCA6) is a neurodegenerative disorder caused by abnormal expansions of a trinucleotide CAG repeat in exon 47 of the CACNA1A gene, which encodes the $\alpha 1 \mathrm{~A}$ subunit of the P/Q-type voltage-gated calcium channel. The CAG repeat expansion is translated into an elongated polyglutamine tract in the carboxyl terminus of the $\alpha 1 \mathrm{~A}$ subunit. The $\alpha 1 \mathrm{~A}$ subunit is the main pore-forming subunit of the P/Q-type calcium channel. Patients with SCA6 suffer from a severe form of progressive ataxia and cerebellar dysfunction. Design of treatments for this disorder will depend on better definition of the mechanism of disease. As a disease arising from a mutation in an ion channel gene, SCA6 may behave as an ion channelopathy, and may respond to attempts
\end{abstract}

to modulate or correct ion channel function. Alternatively, as a disease in which the mutant protein contains an expanded polyglutamine tract, SCA6 may respond to the targets of drug therapies developed for Huntington's disease and other polyglutamine disorders. In this review we will compare SCA6 to other polyglutamine diseases and channelopathies, and we will highlight recent advances in our understanding of $\alpha 1 \mathrm{~A}$ subunits and SCA6 pathology. We also propose a mechanism for how two seemingly divergent hypotheses can be combined into a cohesive model for disease progression. Key Words: Spinocerebellar ataxia type 6 (SCA6), channelopathy, polyglutamine disease, P/Q-type calcium channel, Purkinje cell, cerebellum.

\section{INTRODUCTION}

Spinocerebellar ataxia type six (SCA6) is a progressive ataxic disorder caused by a CAG repeat expansion in the CACNAIA gene encoding the $\alpha 1$ subunit of the neuronal $\mathrm{P} / \mathrm{Q}$-type voltage-gated calcium channel (VGCC). ${ }^{1,2}$ SCA6 is one of three dominantly inherited neurological disorders caused by mutations in the gene encoding the $\alpha 1 \mathrm{~A}$ subunit. ${ }^{3,4}$ SCA6 is also one of 10 polyglutamine diseases caused by expansion of a native, polyglutamine-encoding CAG tract. ${ }^{5-7}$ Thus, based on the affected gene and the mutational mechanism, the pathogenesis of SCA6 may be related to the ion channel disorders (channelopathies) and/or the CAG repeat disorders (polyglutamine diseases). Currently, there is no treatment for this neurodegenerative disease. Successful therapeutic strategies must be targeted to a valid pathological mechanism, and thus understanding the underlying mechanisms of disease is crucial to finding a proper treatment. This review addresses the molecular mechanisms of SCA6 pathogenesis and highlights recent ad-

Address correspondence and reprint requests to: Christopher M. Gomez, MD, Department of Neurology, University of Chicago, $5841 \mathrm{~S}$. Maryland Avenue, MC 2030, Chicago, IL 60637; E-mail: cgomez@ neurology.bsd.uchicago.edu. vances in our understanding of $\alpha 1 \mathrm{~A}$ processing and SCA6 toxicity. We will focus on the relationship of SCA6 to other channelopathies and to other polyglutamine diseases in an attempt to further our understanding of the mechanisms of SCA6 pathogenesis.

\section{PHENOTYPE AND PATHOLOGY}

SCA6 is a neurodegenerative disorder of the cerebellum, characterized by gaze-evoked nystagmus, dysarthria, progressive imbalance, and severe limb coordination. ${ }^{1,2,8,9}$ Some patients with SCA6 experience occasional bouts of vertigo, but there is no muscle weakness or cognitive impairment. ${ }^{10}$ SCA6 is a late onset disorder with an average onset age of 50 years. ${ }^{1,2,11,12}$ The disease usually progresses slowly and does not shorten lifespan, but most patients do become wheelchair bound by their late 60s. The worldwide prevalence is variable, being highest in Japan and moderate in Europe, although accurate absolute numbers are not available. Genetic epidemiologic studies in England have estimated the frequency of the disease-causing mutation at approximately 1 in every 10,000 individuals. ${ }^{13}$

Magnetic resonance imaging of SCA6 patients shows mild to moderate cerebellar atrophy compared with 
healthy brains. ${ }^{9,14}$ Postmortem analysis of SCA6 brains demonstrates cerebellar atrophy due to selective Purkinje cell degeneration. ${ }^{2,9,15,16}$ SCA6 brains display a striking selective loss of Purkinje cells, with particularly high losses in the midline vermal region of the cerebellum. ${ }^{17}$ The surviving Purkinje cells frequently have decreased dendritic arborizations and decreased cytoplasmic organelles compared with controls. ${ }^{17}$ Mild to moderate granule cell loss appears to be secondary to Purkinje cell loss, but basket, stellate, and Golgi cells of the cerebellar cortex appear largely unaffected.

\section{P/Q-TYPE CALCIUM CHANNELS}

SCA6 is caused by a mutation in the C-terminus of the $\alpha 1 \mathrm{~A}$ subunit of the P/Q-type VGCC. P/Q-type calcium channels are comprised of the $\alpha 1 \mathrm{~A}$ pore-forming subunit, and at least two auxiliary subunits, $\beta$ and $\alpha 2 \delta{ }^{18}$ Although the auxiliary subunits do not directly form the channel, they are required for proper channel function. ${ }^{19}$ The $\alpha 1 \mathrm{~A}$ subunit has a structure similar to other $\alpha 1$ subtypes; i.e., four linked homologous domains, each comprised of six transmembrane segments (S1-6). The four linked domains are linked by three intracellular loops (I-II, II-III, and III-IV) and are flanked by cytoplasmic $\mathrm{N}$ and $\mathrm{C}$-termini, all of which interact with a variety of auxiliary subunits and regulatory proteins. The genes encoding the $\alpha 1$ subunits are highly spliced. ${ }^{20}$ Some forms of the $\alpha 1 \mathrm{~A}$ subunit encode a polyglutamine tract that is variable in length, and the expansion of which underlies SCA6. ${ }^{2}$ The $\alpha 1 \mathrm{~A}$ subunit is one of the most widely expressed of all $\alpha 1$ subunits, as it has been found throughout the brain, heart, pituitary, liver, spleen, and kidneys, with particularly prominent expression in Purkinje and granule cells of the cerebellar cortex. ${ }^{21-24}$ The $\alpha 1 \mathrm{~A}$ subunit is responsible for both the slowly inactivating highly agatoxin sensitive $\mathrm{P}$-current as well as the fast inactivating conotoxin sensitive Q-current. ${ }^{25-28}$

As with other $\alpha 1$ subunits, the intracellular C-terminus of the $\alpha 1 \mathrm{~A}$ subunit has been implicated in a number of protein-protein interactions that play a prominent role in modulating channel activity. ${ }^{19}$ Calcium calmodulin readily binds to an EF hand domain in the $\alpha 1 \mathrm{~A} \mathrm{C}$ terminus..$^{29,20}$ This binding inhibits channel currents and alters synaptic efficacy. The C-terminus can also alter channel inactivation kinetics and possibly $\alpha 1 \mathrm{~A}$ subcellular localization. ${ }^{30-32}$ Despite these shared functions, the $\alpha 1 \mathrm{~A}$ subunit differs from other calcium channel $\alpha 1$ subunits primarily in its C-terminus. However, a unique function for the $\alpha 1 \mathrm{~A} \mathrm{C}$-terminus that can account for its divergence from other $\alpha 1 \mathrm{C}$-termini has not been fully elucidated. One functional distinction concerns the $\beta 4$ auxiliary subunit, which selectively binds to the $\alpha 1 \mathrm{~A}$ $\mathrm{C}$-terminus, and upon binding alters channel kinetics. ${ }^{33,34}$ Interestingly, the polyglutamine tract, which is expanded in SCA6, is only present in $\alpha 1 \mathrm{~A}$ subunits, and is not evolutionarily conserved, as polyglutamine tracts are not present in rodent $\alpha 1 \mathrm{~A} \mathrm{C}$-termini. A complete understanding of the function of the C-terminus is imperative because the SCA6 mutation is contained within the distal C-terminus of $\alpha 1 \mathrm{~A}$, and the therapeutic target will invariably affect $\mathrm{C}$-terminal functioning.

\section{CACNA1A GENETICS}

Genetic studies confirm that P/Q-type channels are critical for neuronal function and viability, although the details of the role(s) P/Q-type channels play are unclear and may be complicated. In mice, spontaneous or targeted mutations of this gene typically lead to recessively inherited severe neurologic disorders characterized by ataxia, seizures, and dystonia. ${ }^{4,35-42}$ Mutations of several types, both loss of function and altered function, have roughly similar phenotypes. The molecular consequence of one of these mutations, $\mathrm{Tg}^{(\mathrm{la})}$, is selective loss of the complete C-terminus of the $\alpha 1 \mathrm{~A}$ subunit after either codon 1922 or codon 1967, resulting in seizures, and episodic and progressive cerebellar disease with degeneration of Purkinje cells. ${ }^{37}$ Because in this mouse the mutant "tail-less" P/Q-type channels remain largely functional within the cerebellar neurons, this mutant points to a key role for the C-terminus in cerebellar neuronal function and viability, a fact relevant to the human disease SCA6.

In humans, only mutations exhibiting more pronounced phenotypes, with autosomal dominant inheritance, have been recognized. ${ }^{4}$ Mutations in the CACNA1A gene cause two dominantly inherited episodic disorders in humans; familial hemiplegic migraine $(\mathrm{FHM})^{43}$ and episodic ataxia type 2 (EA-2). ${ }^{44}$ Both are typically caused by point mutations in the CACNA1A gene, and their disease mechanisms have both been linked to altered calcium channel function; i.e., they are channelopathies. Patients with FHM suffer from severe headaches, hemiparesis, or hemianesthenia, and occasionally episodic imbalance, with typical onset beginning at ages 10 to 20 years. ${ }^{43,45}$ In FHM, point mutations at conserved residues in CACNA1A can lead to changes in current densities, altered inactivation kinetics, and altered open probability of P/Q-type channels. ${ }^{41,43,45}$

In EA-2, patients experience episodic imbalance, dysarthria, vertigo, and hand incoordination. CACNAIA mutations found in EA-2 are most commonly nonsense or splicing mutations that result in $\alpha 1 \mathrm{~A}$ subunits that are truncated within the repeat domains, or that predict skipped exons with loss of function of the mutant in expression studies. ${ }^{4,46,47}$ Several studies suggest that the truncated subunits exhibit dominant inheritance by exerting a dominant negative effect on the wild-type P/Q current activity. ${ }^{44,48,49}$ 
SCA6 is a progressive ataxic disorder caused by a trinucleotide repeat (CAG) expansion in exon 47 of the CACNA1A gene. $^{2}$ This repeat is translated into a polyglutamine expansion in the distal C-terminus of certain splice variants of the P/Q-type calcium channel $\alpha 1 \mathrm{~A}$ subunit. The CAG repeat in CACNA1A is polymorphic in length. Healthy individuals normally have between 4 and 18 glutamines, while individuals with SCA6 have between 19 and 33 glutamines. ${ }^{50}$ Age of onset of disease in SCA6 is inversely correlated with the length of the glutamine repeat expansion. For example, individuals with a glutamine repeat of 20 begin to exhibit symptoms in their 70s, while individuals with a repeat length of 30 glutamines begin to exhibit symptoms as early as 30 years old. ${ }^{12}$ The findings of the SCA6 mutation in humans and the spontaneous mouse mutant leaner $\mathrm{C}$ terminal $\alpha 1 \mathrm{~A}$ splice mutation strongly suggest that genetic mutation of the C-terminus has significant functional consequences.

\section{SCA6 AS AN ION CHANNELOPATHY}

The involvement of a functional ion channel subunit $(\alpha 1 \mathrm{~A})$ of a VGCC in SCA6 favors the hypothesis that SCA6 is an ion channel disorder in which the polyglutamine expansion in the $\mathrm{C}$-terminus of $\alpha 1 \mathrm{~A}$ causes ataxia by altering calcium channel function. Numerous studies have attempted to determine how the polyglutamine expansion affects the properties of the P/Q-type calcium channel, and ultimately how a small polyglutamine expansion in a VGCC may lead to cell death. ${ }^{15,16,51-55}$ Unfortunately, these results appear highly variable and depend on which expression system and auxiliary VGCC subunits are used. Nevertheless, all of these studies provide insight into the various aspects of SCA6 pathology, in particular, the basis for the selective Purkinje cell degeneration in SCA6.

The first group to analyze the effect of expanded polyglutamine tracts on P/Q-type calcium channel functioning reported only moderate alterations in channel inactivation kinetics. ${ }^{52}$ In BHK cells stably expressing $\alpha 2 \delta$ and $\beta 1 \mathrm{a}$, an $8-\mathrm{mV}$ hyperpolarizing shift in the voltage dependence of inactivation was found in cells expressing expanded (Q30 or Q40) $\alpha 1 \mathrm{~A}$ compared with unexpanded controls (Q4) ${ }^{52}$ However, the disease-causing expansion of Q24 did not alter kinetics in this model. In this study, no changes in current density and only a slightly hyperpolarizing shift in the voltage dependence of activation in Q30 and Q40 containing calcium channels were observed. The researchers hypothesized that the shift in inactivation kinetics decreases the number of channels available at resting membrane potential, and thus decreases the amount of calcium entering the cell. In these same cells, apoptotic cell death was analyzed using the nicked DNA staining assay, TUNEL. Even though ki- netic alterations were observed, none of the cells transfected with the expanded (Q30 or Q40) calcium channels displayed TUNEL staining, indicating that in this system these kinetic alterations were not sufficient to induce apoptosis.

In another study, Toru et al. ${ }^{55}$ had detected an effect using the Q24 allele in HEK cells cotransfected with $\alpha 2 \delta, \beta 1 \mathrm{a}$, and $\alpha 1 \mathrm{AQ} 24$, recording a 6-mV hyperpolarizing shift in the voltage dependence of inactivation compared with unexpanded controls (Q13). Similarly, cells expressing Q28-containing calcium channels exhibited an $11-\mathrm{mV}$ hyperpolarizing shift, greater than the 8-mV shift previously reported in calcium channels with much larger expansions (Q30 and Q40). ${ }^{52}$ Interestingly, the negative shifts in inactivation kinetics were only observed in splice variants of the P/Q-type calcium channel that contain exon 31 , which encode the residues asparagine and proline $(\mathrm{NP}+)$. Calcium channels that do not contain the NP residues (NP-) exhibit a positive shift in the voltage dependence of inactivation when the channel contains an expansion of Q28. The studies from these groups are consistent with the view that the changes in the polyglutamine-expanded $\mathrm{NP}+\alpha 1 \mathrm{~A}$ calcium channels will result in a decrease in calcium influx into the cell.

Several investigators have explored whether different subtypes of beta auxiliary VGCC subunits determine the channel gating consequences of $\alpha 1 \mathrm{~A}$ subunits bearing SCA6 polyglutamine expansions. Our group studied $\alpha 1 \mathrm{~A}$ subunit alleles expressed in Xenopus oocytes by injecting expanded (Q22, Q26, or Q30) or unexpanded (Q13) $\alpha 1 \mathrm{~A}$ subunits together with $\alpha 2 \delta$ and $\beta 2,3$, or 4 subunits. We found that expression of the Q30 expanded $\alpha 1 \mathrm{~A}$ led to a hyperpolarizing shift in the voltage dependence of activation and a delay in inactivation, but only when oocytes were expressing expanded $\alpha 1 \mathrm{~A}$ and $\beta 4$ subunits. ${ }^{16} \mathrm{~A}$ trend for similar effects was seen in Q20or Q26-expressing oocytes. No significant changes were observed in cells expressing $\alpha 1 \mathrm{~A}$ with $\beta 2$ or $\beta 3$.

The influence of beta subunit subtype was also seen in G-protein coupling, which is determined in part by Cterminal motifs. Because the splice variant used in these studies was sensitive to G-protein mediated inactivation, we co-expressed the G-protein coupled $\mu$-opioid receptor, together with unexpanded $\alpha 1 \mathrm{~A}, \alpha 2 \delta$, and $\beta 2,3$, or 4 and treated with DAMGO to inhibit inactivation. This inhibition was attenuated in cells expressing Q26 and $\mathrm{Q} 30$ expanded $\alpha 1 \mathrm{~A}$ and $\beta 4$, suggesting that the delay in inactivation and the prevention of G-protein mediated inhibition of inactivation would lead to an increase in calcium influx. ${ }^{16}$ These results suggest a model in which polyglutamine-mediated $\alpha 1 \mathrm{~A}$ alterations are dependent on the $\beta$ subunit and result in an increase in intracellular calcium. Surprisingly, these findings are contradictory to those of the two previous studies, ${ }^{52,55}$ and suggest 
that polyglutamine-expanded SCA6-associated P/Q-type channels lead to increased calcium entry into cerebellar neurons.

A fourth study reported that HEK cells stably expressing $\beta 1$ and $\alpha 2 \delta$ exhibited an increase in the surface expression of $\alpha 1 \mathrm{~A}$ containing Q23, Q27, or Q72 repeats compared with unexpanded controls (Q4 and Q11). The increase in surface expression resulted in a twofold increase in current density but did not alter channel kinetics. ${ }^{54}$ These changes occurred without an apparent change in mRNA levels, suggesting that the increase in current density was due to increased stability of polyglutamine-expanded $\alpha 1 \mathrm{~A}$ subunits. Other proteins bearing expanded polyglutamine tracts, such as huntingtin, have shown similar increases in stability and resistance to degradation relative to the unexpanded proteins. ${ }^{56-59}$ The relevance of these studies to SCA6 is questionable because the expansion Q72 in $\alpha 1 \mathrm{~A}$ greatly exceeds the largest allele identified in SCA6, Q33. However, current density was increased relatively equally in all expanded $\alpha 1 \mathrm{~A}$ expressing cells (Q23, Q27, and Q72).

In a follow-up study the same investigators expressed expanded Q23 and Q72, unexpanded, Q11 $\alpha 1 \mathrm{~A}$ in HEK cells transiently, together with $\beta 2 \mathrm{a}$ or $\beta 4$ auxiliary subunits, rather than $\beta 1$ subunits. ${ }^{60}$ Interestingly, in this experiment no changes in current densities were observed. This finding illustrates either the importance of $\beta$ subunit subtype or the difference between transient or stable expression of auxiliary subunits. However, in the $\beta 2 \mathrm{a}$ or $\beta 4$ studies the expanded polyglutamine tract did shift the steady state inactivation in the positive direction by $6 \mathrm{mV}$. In addition, expanded polyglutamine tracts facilitated a decrease in activity-dependent inactivation, but not inactivation or activation of current kinetics. For example, after a $100 \mathrm{~Hz}$ pulse, 54\% of the current in unexpanded $\alpha 1 \mathrm{~A}$-expressing cells was inactivated where only $46 \%$ inactivation occurred in expanded (Q72) $\alpha 1 \mathrm{~A}$ expressing cells. These properties again favored increased calcium entry into SCA6 neurons.

\section{Conclusion}

In all instances the $\alpha 1 \mathrm{~A}$ subunits expressing SCA6 mutations can form functioning channels. Electrophysiologic analysis of the channel function indicates that the expanded glutamine tracts alter channel kinetics and functioning. From these studies, it is clear that different model systems can affect the channel kinetics and may account for some of the differences among experiments. However, more importantly, splice forms of $\alpha 1 \mathrm{~A}$ and the different $\beta$ subunit forms appear to account for some significant differences in findings among groups and even within individual studies. This may have realistic implications, as $\alpha 1 \mathrm{~A}$ splice forms and the various forms of $\beta$ subunits all display divergent, cell-specific expression patterns in vivo. These observations suggest that a lethal combination of P/Q-type channel variables yet to be completely specified may contribute to pathogenesis.

Although the model systems used are very useful in controlling for many variables, they may be too simple to model the complexity of the biophysical properties of the cerebellum. These changes may be ideally studied using direct cerebellar slice recordings, where proper ratios of $\beta$ subunits, $\alpha 1 \mathrm{~A}$ splice forms, and activity can be maintained after genetic manipulation has been carried out through transfection methods. These types of studies need to be adequately assessed prior to utilizing therapeutics directed at altering channel kinetics. As the literature stands, it appears that SCA6 mutations can both increase and decrease the amount of calcium entering the cell, depending upon the model system used. This important distinction needs to be addressed in the proper adequately complex system.

\section{SCA6 AS A POLYGLUTAMINE DISEASE}

The mutational mechanism in SCA6 suggests that the pathogenesis of this disorder may share features in common with the group of disorders known as the polyglutamine diseases. Huntington's disease (HD), Machado-Joseph's disease (MJD/SCA3), spinocerebellar ataxias (SCA) 1, 2, 7, and 17, dentatorubropallidoluysian atrophy, and spinal and bulbar muscular atrophy (SMBA) are all caused by abnormal expansion of a polyglutamine tract normally occurring in a set of unrelated proteins. In each of these disorders a protein containing a normal stretch of glutamines (in healthy individuals, typically between 10 and 40 glutamines in length) is abnormally elongated, typically to 40 to 100 glutamines. ${ }^{12,61-63}$ Several of these disorders, particularly Huntington's disease, have been extensively studied, and numerous therapeutic approaches have been explored in experimental models and in early clinical trials. If SCA6 is mechanistically similar it may benefit from therapeutic advances made in these polyglutamine diseases.

With the exception of SMBA, an X-linked disorder, all polyglutamine diseases exhibit an autosomal dominant pattern of inheritance (i.e., only one mutant allele is required for the disease phenotype to develop). In addition, in all polyglutamine diseases the size of the glutamine repeat expansion varies between some individuals and the age of onset of disease symptoms is inversely correlated with the size of the repeat expansion. ${ }^{12,61,62}$ Furthermore, in subsequent generations the expanded glutamine tract is susceptible to further expansion, favoring an even earlier age of onset. ${ }^{63-65}$ This clinical and genetic evidence suggests that polyglutamine diseases are caused by a "toxic gain-of-function." As a prerequisite to the design of rational therapies, molecular and cellular studies have subsequently sought to understand 
the potentially common structural and functional basis for the pathogenesis of the selective neuronal degeneration in the polyglutamine diseases. One common feature has been that the affected proteins in HD, SMBA, SCA3, and SCA7 all appear to be proteolytically processed at discrete sites within the proteins in both the normal and disease-causing forms. ${ }^{66-69}$ Moreover, all polyglutamine disease-causing proteins (except ataxin-2) form nuclear aggregates. ${ }^{5,6}$ These commonalities and other common pathways of processing in the nucleus may reveal powerful therapeutic strategies.

Although many similarities exist between the polyglutamine diseases, SCA6 differs from most others in this class in several respects. First, SCA6 is caused by an expanded repeat that is "pathological" at a range of 19 to 33 glutamines. This tract length is well within the range of healthy polyglutamine tracts in other diseases that cause neurodegeneration only when they contain greater than 35 glutamines. Studies of "naked" polyglutamine tracts (i.e., those that are not part of the endogenous polypeptide where they occur) conventionally use repeats of 35 to 45 glutamines as their negative control, and report no cell death above baseline. Therefore, polyglutamine tracts that are part of the $\alpha 1 \mathrm{~A}$ subunit protein are toxic to cells at much lower sizes.

Second, until recently SCA2 and SCA6 appeared to share common features, distinguishing them from the other SCAs. SCA2 and SCA6 are the only polyglutamine disease proteins that do not form ubiquitinated nuclear aggregates. In all other polyglutamine diseases except SCA2 and SCA6, the accumulation of the diseasecausing polyglutamine tract in the nucleus was necessary to mediate the disease. ${ }^{56,67,70-83}$ In the case of SCA2, the disease appears to be mediated by an effect on protein translation. ${ }^{76,84}$ In the case of SCA6, as discussed above, the expanded polyglutamine tract occurs in a neuronal ion channel protein, whose function may be altered by the expanded tract. Third, clinical genetic studies point to a role played by the polyglutamine tract in the $\alpha 1 \mathrm{~A}$ subunit in modulating the severity of SCA2 ${ }^{85}$ Specifically, SCA2 patients with larger normal, but not SCA6causing, polyglutamine tracts in the $\alpha 1 \mathrm{~A}$ gene appear to have earlier onset ataxia than those with small $\alpha 1 \mathrm{~A}$ polyglutamine tracts.

\section{C-Terminal fragments}

Recent studies, coupled with other unexplained observations, have provided stronger support for a pathogenic mechanism in SCA6 that more closely resembles the other polyglutamine diseases and have provided evidence against the absolute role of P/Q-type channel kinetics in disease pathogenesis.

Over a decade ago, it was reported ${ }^{86}$ that the C-terminus of the $\alpha 1 \mathrm{C}$ voltage-gated calcium channel is cleaved by calpain, a calcium-dependent protease, from the full-length protein in skeletal muscle. This cleavage phenomenon in hippocampal cultures is facilitated by activity of accompanying NMDA receptors and results in a fourfold increase in current density. ${ }^{87}$ Also, C-terminal fragments of $\alpha 1 \mathrm{~A}$ subunits have been detected in protein extracts of purified $\alpha 1 \mathrm{~A}$ and $\alpha 1 \mathrm{~A}$-expressing cells. ${ }^{88}$ While initially seeming to have little importance, these fragments took on greater significance when the SCA6 mutation was identified in the extreme distal $\mathrm{C}$-terminus of the $\alpha 1 \mathrm{~A}$ subunit.

Kubodera et al. ${ }^{51}$ recently reported that the C-terminus of the $\alpha 1 \mathrm{~A}$ subunit is post-translationally processed and cleaved from the full-length protein. This is consistent with reports of $\alpha 1 \mathrm{C}$ subunits in which the $\mathrm{C}$-terminus is also cleaved near the same region. ${ }^{86}$ This study was notable because of the observation that the $\alpha 1 \mathrm{~A} \mathrm{C}$ terminal fragments were only detected in protein extracts from HEK cells transiently expressing expanded (Q30) $\alpha 1 \mathrm{~A}$, although it contrasted with the previous findings on C-terminal cleavage. These investigators ${ }^{51}$ hypothesized that both the expanded and unexpanded $\alpha 1 \mathrm{~A}$ subunits were cleaved, but that the expanded C-terminus was selectively resistant to degradation. This property has been observed in other polyglutamine proteins. More significantly, these investigators ${ }^{51}$ found that in HEK cell cultures expressing C-terminal fragments cell death, as determined by dye exclusion, was increased in an alleledependent manner. C-termini containing Q28 were significantly more toxic than C-termini harboring Q13. In this experiment there was no difference in toxicity of full-length $\alpha 1 \mathrm{~A}$ subunits bearing wild-type or expanded polyglutamine tracts.

Recently our group has confirmed the cleavage of the $\alpha 1 \mathrm{~A}$ subunit within cerebellar tissue. ${ }^{89}$ Of greater significance, however, was our observation that the endogenous free C-terminal fragment localizes to Purkinje cell nuclei. In addition, C-terminal fragments cleaved from recombinant $\alpha 1 \mathrm{~A}$ subunits or expressed as isolated Ctermini localize to nuclei in cultured cell lines and primary granule cell cultures. Interestingly, using immunoblotting to estimate protein abundance, we found that cleavage and nuclear localization are not affected by polyglutamine length. The expanded and unexpanded C-terminal fragment was found in equal abundance in HEK cell cultures transiently transfected with full-length $\alpha 1 \mathrm{~A}$ subunits.

To understand the molecular basis for the nuclear translocation, our group has characterized three potential nuclear localization signals (NLSs) to determine whether they target the C-terminal fragment to the nucleus. In HEK cell cultures, elimination of any of the three NLS reduces nuclear localization of the C-terminus, but elimination of all three NLS drastically reduces C-terminal nuclear localization. ${ }^{89}$ This redundancy substantiates the importance of nuclear localization of the C-terminus. Interestingly, none of the NLS sequences present in the 
C-terminus conform to the classic structure of an NLS. There are two recognized classes of NLS; monopartite, which contain a string of basic residues, and bipartite, which consist of two monopartite NLS separated by twelve or more residues. ${ }^{90-92}$ In the $\alpha 1 \mathrm{~A}$ subunit, the motifs participating in nuclear localization, referred to as NLS1, 2, 3, are separated by at least 66 residues; too great a distance to form bipartite NLSs. Moreover, the $\alpha 1 \mathrm{~A}$ NLS1, comprised of the amino acids PKARRLD, is the strongest of the NLSs tested. This motif does not conform to a monopartite NLS because the string of basic residues is interspersed with the nonpolar residues Ala, Pro, and Leu. However, the $\alpha 1 \mathrm{~A}$ NLS1 actually resembles an emerging class of NLSs, like those found in c-myc (PAAKRVKLD). ${ }^{90}$ Although we were unable to completely abolish nuclear targeting with mutation to a single NLS, the strength of the $\alpha 1 \mathrm{~A}$ NLSs and nuclear targeting suggest an important function of the $\mathrm{C}$-terminus in the nucleus.

Although the purpose of cleavage and nuclear translocation of the $\alpha 1 \mathrm{~A} \mathrm{C}$ terminus has yet to be explained, it is likely to be involved in a nuclear signaling event, perhaps relating to gene expression. Recent evidence demonstrating that the $\mathrm{C}$-terminal fragment of the $\alpha 1 \mathrm{C}$ subunit of the L-type calcium channel acts as a transcription factor in cell nuclei, ${ }^{93}$ suggests a similar role for $\alpha 1 \mathrm{~A} \mathrm{C}$-terminal fragments in cell nuclei. Interestingly, many polyglutamine disease proteins are known to interact with transcription factors and may be transcriptional regulatory elements themselves.

Our group has also studied the toxicity of the $\alpha 1 \mathrm{~A}$ C-terminal fragment. We demonstrated that in HEK and primary granule cell cultures, expression of the $\alpha 1 \mathrm{~A}$ C-terminal fragment, when bearing an expanded polyglutamine tract of 33 glutamines, is toxic to cells. ${ }^{89}$ This toxicity is independent of the function of the full-length protein. In addition, like other polyglutamine diseases the toxicity is highly dependent on the nuclear localization of the expanded protein. Exclusion of the $\mathrm{C}$-terminal fragment from the nucleus by mutating the threeconserved NLSs decreases toxicity (as analyzed by propidium iodide exclusion) in HEK cells stably expressing $\beta 3$ and $\alpha 2 \delta$. This is similar to the finding that exclusion of expanded huntingtin from the nucleus prevents cell death, ${ }^{80}$ and exclusion of expanded ataxin- 1 by mutation of the NLS in SCA1 mice prevents cell death and ataxic phenotypes. ${ }^{94}$ Attachment of a nuclear export signal (NES) to an expanded androgen receptor, the cause of SMBA, inhibits toxicity in drosophila models. ${ }^{95}$ Conversely, atrophin-1, the protein mutated in dentatorubropallidoluysian atrophy, is localized to the cytoplasm and the nucleus. However, removal of the NES results in the polyglutamine tract accumulating in the nucleus. ${ }^{77}$ Given that the SCA6 expanded C-terminal fragment is the first demonstration that a small CAG expansion of only 28 or
33 repeats can be toxic to cells, these data suggest that SCA6 may have a similar disease mechanism as other polyglutamine diseases. Thus, treatments directed against proteolysis, nuclear accumulation, or nuclear entry might provide a useful treatment for SCA6, in addition to the other polyglutamine diseases.

\section{Conclusion}

These recent findings have two important implications concerning disease pathogenesis. First, because the cleaved C-terminus of the $\alpha 1 \mathrm{~A}$ subunit contains a polyglutamine tract that is expanded in SCA6 and is translocated to the nucleus, they suggest that SCA6 shares disease mechanisms in common with other polyglutamine diseases, such as HD, and may share potential treatment strategies. Second, because this C-terminal cleavage eliminates the $\mathrm{C}$-terminus from the functioning $\alpha 1 \mathrm{~A}$ subunit, this observation has important implications for the interpretation of previous electrophysiologic studies. It is conceivable that $\mathrm{C}$-terminal cleavage effects kinetic functioning of the full-length channel and that C-terminal cleavage may occur differently in different cell types. Because traditionally, whole cell patch-clamp techniques were used and not individual channel recording, it is imperative to determine, in the expression systems used to study the function of the polyglutamineexpanded $\alpha 1 \mathrm{~A}$ subunit, if the $\mathrm{C}$-terminus is cleaved, and to what extent. Thus, the ratio of cleaved to uncleaved $\alpha 1 \mathrm{~A}$ subunits in previous P/Q-type channel recordings, and the affect of cleavage on normal P/Q-type channel kinetics should be determined to assess the relative contributions of the two mechanisms.

\section{POLYGLUTAMINE-MEDIATED TOXICITY}

Given recent findings that the polyglutamine-containing C-terminal fragments may be toxic in an alleledependent manner, it is interesting to speculate how prior kinetic data shall be interpreted based on the finding of endogenous cleavage of $\alpha 1 \mathrm{~A}$ subunits. Although the kinetic data have yet to be directly linked to toxicity, some studies have reported death in cells expressing SCA6 mutations in full-length ion-conducting $\alpha 1 \mathrm{~A}$ subunits. In one study, HEK cells expressing full-length $\alpha 1 \mathrm{~A}$ subunits bearing a glutamine expansion of Q28 exhibited signs of apoptotic cell death in a qualitative nicked DNA assay. ${ }^{15}$ Another group failed to detect an apoptotic effect in cells expressing $\alpha 1 \mathrm{~A}$ with Q24, Q30, or Q40 repeats. ${ }^{52}$ The same group later reported that in serumstarved conditions, the expanded $\alpha 1 \mathrm{~A}$ was more toxic than its unexpanded counterparts. ${ }^{53}$ However, the expanded $\alpha 1 \mathrm{~A}$ failed to kill cells under normal growth conditions. It was concluded that the polyglutamine expansion prevents the $\alpha 1 \mathrm{~A}$ from performing its protective functions as $\alpha 1$ activity is required for survival. ${ }^{96,97}$ This 
intriguing loss-of-function model has yet to be replicated.

The discrepancies in these reports may be explained by studies demonstrating that the toxicity of the $\alpha 1 \mathrm{~A}$ C-terminus correlates with the extent of nuclear localization (i.e., demonstrating dose dependence of nuclear localization on cell death). ${ }^{89}$ The altered culture conditions for full-length $\alpha 1 \mathrm{~A}$ subunits may affect cleavage or nuclear localization. Similarly, the amount of C-terminus accumulating in the nucleus may vary among different cell types. If this is the case, full-length $\alpha 1 \mathrm{~A}$ may sequester the $\mathrm{C}$-terminus and prevent nuclear entry and subsequent toxicity.

\section{Purkinje cell selectivity in SCA6}

In SCA6 and some of the other forms of dominantly inherited SCA, Purkinje cells are lost with markedly selective sparing of other neuronal types. While prominent death of Purkinje cells in multiple forms of SCA may point to a selective vulnerability of Purkinje cells to certain neuronal stresses, the abundant expression of the $\alpha 1 \mathrm{~A}$ subunit in the cerebellum compared with other regions of the nervous system may explain part of the neuronal selectivity. Curiously, the $\alpha 1 \mathrm{~A}$ subunit is also highly expressed in cerebellar granule cells, which are not affected in SCA6. However, CACNA1A, the gene encoding the $\alpha 1 \mathrm{~A}$ subunit, is highly alternatively spliced. ${ }^{20}$ The splice variant encoding the polyglutamine expansion contains the complete exon $47 .^{2}$ Studies have shown that this splice variant appears in abundance only in cerebellar Purkinje cells. ${ }^{15,16}$ The reverse transcriptasepolymerase chain reaction analysis of exon 47 of the CACNA1A gene confirmed that the cerebellum expressed 10 to 20 times more exon 47 than the cortex, thalamus, or hippocampus and Purkinje cell bodies displayed the highest levels of staining after in situ hybridization to the exon 46-47 boundary. ${ }^{15}$ Moreover, using an $\alpha 1 \mathrm{~A} \mathrm{C}$ terminus-specific antibody, a significant proportion of the cleaved C-terminus is detected in Purkinje cell nuclei, while no $\mathrm{C}$-terminus is detected in granule cell nuclei. ${ }^{89}$ Splice variants encoding shorter forms of the $\alpha 1 \mathrm{~A}$ subunit are more common in other neuronal types. This suggests that splice variants containing the polyglutamine expansion, as in SCA6, are selectively present in Purkinje cells.

Polyglutamine-dependent alterations in ion channel kinetics have also been linked to alternative splice forms of $\alpha 1 \mathrm{~A}$. For example, in one study the polyglutaminemediated alterations in calcium channel kinetics were observed only in cells expressing $\alpha 1 \mathrm{~A}$ subunits that contain the residues $\mathrm{NP}(\mathrm{NP}+)$. The $\alpha 1 \mathrm{~A}$ subunits that did not contain the NP residues (NP-) were not affected by polyglutamine expansions. ${ }^{55}$ Interestingly, Purkinje cells contain primarily $\mathrm{NP}+\alpha 1 \mathrm{~A}$ while granule cells contain both $\mathrm{NP}-$ and $\mathrm{NP}+\alpha 1 \mathrm{~A}$, leading to the suggestion that expression of the NP-variant may explain the sparing of granule cells from polyglutamine toxicity. ${ }^{55}$ Similarly, studies demonstrating altered P/Q-type calcium channel gating induced by $\alpha 1 \mathrm{~A}$ subunits bearing SCA6 mutations only with Purkinje cell-specific $\alpha 1 \mathrm{~A}$ splice forms and subunit combinations may in part explain the selectivity of cell death. ${ }^{16}$ Taken together, these data suggest that a combination of alternative splicing and preferential expression of certain auxiliary subunits, and possibly factors involved in generation and nuclear localization of the C-terminal fragment, may determine Purkinje cell toxicity.

\section{MULTIMODAL HYPOTHESIS}

Presently, from the standpoint of a model for disease pathogenesis, there is experimental evidence that indicates that SCA6 exhibits features of both a "channelopathy" and a "polyglutamine disease." The polyglutamine expansion is toxic to cells independent of the full-length channel protein, but apparently only when cleaved from the full-length channel. ${ }^{51,89}$ In addition, the SCA6 mutation alters the kinetics and functioning of the P/Q-type calcium channel, although the ultimate effect of $\alpha 1 \mathrm{~A}$ splice variants and subunit combinations on this change must still be elucidated. Given the prominent role of $\mathrm{P} / \mathrm{Q}$-type calcium channels in cerebellar processing, and that a number of other CACNA1A mutations can cause cerebellar dysfunction without cell loss, the kinetic alterations seen in SCA6 mutations are likely to result in altered cerebellar function.

It is intriguing to speculate that these two properties of SCA6 mutated $\alpha 1 \mathrm{~A}$ subunits may combine to give rise to SCA6. For example, it may be that changes in channel kinetics are responsible for the onset of disease and the initial symptoms observed in patients, while the accumulation and presence of the C-terminal fragment in Purkinje cell nuclei is responsible for the neurodegeneration and the progression of the disease. In support of this is the observation that many patients with SCA6 report a long history of episodic symptoms of ataxia and vertigo prior to developing progressive disease. Many have received a previous diagnosis of Meniere's disease, as is the case for EA-2, another CACNA1A disorder associated with impaired channel function. Disease progression in SCA6 presumably occurs as a result of the effect of the toxic C-terminus.

Similar mechanisms involving a combination of cell dysfunction and cell death have been proposed for other neurodegenerative diseases. For example, transgenic mouse models of HD and SCA1 manifest symptoms either prior to, or in the absence of, neuronal death. ${ }^{98,99}$ Similarly, in Alzheimer's disease patients, synaptic dysfunction, which correlates with cognitive impairment, precedes neurodegeneration and cell loss. ${ }^{100,101}$ Further 
studies are needed for all of these diseases to ascertain the role of the mutant gene products in cellular dysfunction and cell death.

Another intriguing possibility is that alterations in channel kinetics caused by the polyglutamine expansion may lead to altered cleavage or processing of the Cterminus in vivo. Although cell culture experiments suggest that cleavage is not affected by polyglutamine length ${ }^{89}$ until tested directly in Purkinje cells this hypothesis cannot be discarded. Paralleling this notion is the possibility that calcium-activated proteases are responsible for C-terminal cleavage and thus accumulation of the C-terminus in nuclei. Preliminary evidence suggests that calcium and calcium-dependent proteases are not involved in generation of the C-terminal fragment (Kordasiewicz, unpublished results). However, these experiments are limited to the protease inhibitors and calcium chelators currently available, and do not take into account processing that may have a highly intracellular localization. Similarly, it has yet to be determined if calcium-dependent proteases are involved in degradation of the C-terminal product. Interestingly, the C-terminus contains a PEST site, which is a general protease recognition site that has a high affinity for calcium-dependent proteases, in particular calpain. Future experiments should address the mechanisms of generation and stability of the C-terminal fragment in Purkinje cells, and how these mechanisms relate to changes in calcium channel activity.

\section{CONCLUSION}

It is clear that the polyglutamine expansion in SCA6 can alter P/Q-type calcium channel kinetics. Furthermore, these results suggest that the selective Purkinje cell degeneration in SCA6 may be attributed to the differential expression of SCA6 splice forms, auxiliary subunits, and possibly posttranslational modifiers. It is likely this lethal combination of effectors contributes to the selective loss of cerebellar function and Purkinje cell degeneration. This is potentially beneficial from a therapeutic standpoint, as having multiple effectors may allow multiple targets for intervention. However, until a realistic model system (such as a transgenic mouse model) is derived, the ideal approach will likely remain unclear.

In light of the recent discoveries of C-terminal cleavage and nuclear translocation, the mechanism for SCA6 pathogenesis appears even more complicated than initially anticipated. Important questions such as how the glutamine expansion affects channel kinetics, proteolysis, other posttranslational processing, transport, and localization of the $\alpha 1 \mathrm{~A}$ subunit will need to be answered. This unpredicted event may help explain the variability in the kinetic data generated thus far, particularly given that the C-terminus appears to be processed in some of the systems used for the electrophysiologic studies. Potentially, all mechanisms proposed thus far are working concurrently to cause disease in SCA6, but interestingly, may be working at different stages of disease. If this is the case, therapeutics targeted at either channelopathy or polyglutamine disease mechanisms may be most effective at different stages of disease.

\section{REFERENCES}

1. Ishikawa K, Tanaka H, Saito M, et al. Japanese families with autosomal dominant pure cerebellar ataxia map to chromosome 19p13.1-p13.2 and are strongly associated with mild CAG expansions in the spinocerebellar ataxia type 6 gene in chromosome 19p13.1. Am J Hum Genet 1997;61:336-346.

2. Zhuchenko O, Bailey J, Bonnen P, et al. Autosomal dominant cerebellar ataxia (SCA6) associated with small polyglutamine expansions in the alpha $1 \mathrm{~A}$-voltage-dependent calcium channel. Nat Genet 1997;15:62-69.

3. Garg MK, Sanchette PC. Ion channels and channelopathy. J Assoc Physicians India 1999;47:436-439.

4. Pietrobon D. Calcium channels and channelopathies of the central nervous system. Mol Neurobiol 2002;25:31-50.

5. La Spada AR, Taylor JP. Polyglutamines placed into context. Neuron 2003;38:681-684.

6. Orr HT. Beyond the Qs in the polyglutamine diseases. Genes Dev 2001;15:925-932.

7. Ross CA. Polyglutamine pathogenesis: emergence of unifying mechanisms for Huntington's disease and related disorders. Neuron 2002;35:819-822.

8. Garcia-Planells J, Cuesta A, Vilchez JJ, Martinez F, Prieto F, Palau F. Genetics of the SCA6 gene in a large family segregating an autosomal dominant "pure" cerebellar ataxia. J Med Genet 1999;36:148-151.

9. Gomez CM, Thompson RM, Gammack JT, et al. Spinocerebellar ataxia type 6: gaze-evoked and vertical nystagmus, Purkinje cell degeneration, and variable age of onset. Ann Neurol 1997;42: 933-950.

10. Jen JC, Yue Q, Karrim J, Nelson SF, Baloh RW. Spinocerebellar ataxia type 6 with positional vertigo and acetazolamide responsive episodic ataxia. J Neurol Neurosurg Psychiatry 1998;65: 565-568.

11. Geschwind DH, Perlman S, Figueroa KP, Karrim J, Baloh RW, Pulst SM. Spinocerebellar ataxia type 6. Frequency of the mutation and genotype-phenotype correlations. Neurology 1997;49: 1247-1251.

12. van de Warrenburg BP, Hendriks H, Durr A, et al. Age at onset variance analysis in spinocerebellar ataxias: a study in a DutchFrench cohort. Ann Neurol 2005;57:505-512.

13. Craig K, Keers SM, Archibald K, Curtis A, Chinnery PF. Molecular epidemiology of spinocerebellar ataxia type 6. Ann Neurol 2004;55:752-755.

14. Satoh JI, Tokumoto H, Yukitake M, et al. Spinocerebellar ataxia type 6: MRI of three Japanese patients. Neuroradiology 1998;40: 222-227.

15. Ishikawa K, Fujigasaki H, Saegusa H, et al. Abundant expression and cytoplasmic aggregations of [alpha]1A voltage-dependent calcium channel protein associated with neurodegeneration in spinocerebellar ataxia type 6. Hum Mol Genet 1999;8:11851193.

16. Restituito S, Thompson RM, Eliet J, et al. The polyglutamine expansion in spinocerebellar ataxia type 6 causes a beta subunitspecific enhanced activation of P/Q-type calcium channels in Xenopus oocytes. J Neurosci 2000;20:6394-6403.

17. Yang Q, Hashizume Y, Yoshida M, et al. Morphological Purkinje cell changes in spinocerebellar ataxia type 6. Acta Neuropathol (Berl) 2000;100:371-376.

18. Catterall WA. Structure and regulation of voltage-gated $\mathrm{Ca} 2+$ channels. Annu Rev Cell Dev Biol 2000;16:521-555. 
19. Walker D, De Waard M. Subunit interaction sites in voltagedependent $\mathrm{Ca} 2+$ channels: role in channel function. Trends Neurosci 1998;21:148-154.

20. Soong TW, DeMaria CD, Alvania RS, et al. Systematic identification of splice variants in human P/Q-type channel alpha1(2.1) subunits: implications for current density and $\mathrm{Ca} 2+$-dependent inactivation. J Neurosci 2002;22:10142-10152.

21. Hillman D, Chen S, Aung TT, Cherksey B, Sugimori M, Llinas RR. Localization of P-type calcium channels in the central nervous system. Proc Natl Acad Sci U S A 1991;88:7076-7080.

22. Kulik A, Nakadate K, Hagiwara A, et al. Immunocytochemical localization of the alpha 1A subunit of the P/Q-type calcium channel in the rat cerebellum. Eur J Neurosci 2004;19:2169-2178.

23. Mintz IM, Adams ME, Bean BP. P-type calcium channels in rat central and peripheral neurons. Neuron 1992;9:85-95.

24. Westenbroek RE, Sakurai T, Elliott EM, et al. Immunochemical identification and subcellular distribution of the alpha 1A subunits of brain calcium channels. J Neurosci 1995;15:6403-6418.

25. Llinas RR, Sugimori M, Cherksey B. Voltage-dependent calcium conductances in mammalian neurons. The P channel. Ann N Y Acad Sci 1989;560:103-111.

26. Mori Y, Friedrich T, Kim MS, et al. Primary structure and functional expression from complementary DNA of a brain calcium channel. Nature 1991;350:398-402.

27. Randall A, Tsien RW. Pharmacological dissection of multiple types of $\mathrm{Ca} 2+$ channel currents in rat cerebellar granule neurons. J Neurosci 1995;15:2995-3012.

28. Snutch TP, Reiner PB. Ca2+ channels: diversity of form and function. Curr Opin Neurobiol 1992;2:247-253.

29. DeMaria CD, Soong TW, Alseikhan BA, et al. Calmodulin bifurcates the local $\mathrm{Ca} 2+$ signal that modulates P/Q-type $\mathrm{Ca} 2+$ channels. Nature 2001;411:484-489.

30. Lee A, Wong ST, Gallagher D, et al. Ca2+/calmodulin binds to and modulates P/Q-type calcium channels. Nature 1999;399:155-159.

31. Krovetz HS, Helton TD, Crews AL, Horne WA. C-Terminal alternative splicing changes the gating properties of a human spinal cord calcium channel alpha 1A subunit. J Neurosci 2000; 20:7564-7570.

32. Maximov A, Sudhof TC, Bezprozvanny I. Association of neuronal calcium channels with modular adaptor proteins. J Biol Chem 1999;274:24453-24456.

33. Furukawa T, Nukada T, Mori Y, et al. Differential interactions of the $\mathrm{C}$ terminus and the cytoplasmic I-II loop of neuronal $\mathrm{Ca} 2+$ channels with G-protein alpha and beta gamma subunits. I. Molecular determination. J Biol Chem 1998;273:17585-17594.

34. Walker D, Bichet D, Campbell KP, De Waard M. A beta 4 isoform-specific interaction site in the carboxyl-terminal region of the voltage-dependent $\mathrm{Ca} 2+$ channel alpha 1A subunit. J Biol Chem 1998;273:2361-2367.

35. Doyle J, Ren X, Lennon G, Stubbs L. Mutations in the Cacnl1a4 calcium channel gene are associated with seizures, cerebellar degeneration, and ataxia in tottering and leaner mutant mice. Mamm Genome 1997;8:113-120.

36. Fletcher CF, Frankel WN. Ataxic mouse mutants and molecular mechanisms of absence epilepsy. Hum Mol Genet 1999;8:19071912.

37. Fletcher CF, Lutz CM, O'Sullivan TN, et al. Absence epilepsy in tottering mutant mice is associated with calcium channel defects. Cell 1996;87:607-617.

38. Fletcher CF, Tottene A, Lennon VA, et al. Dystonia and cerebellar atrophy in Cacnala null mice lacking P/Q calcium channel activity. FASEB J 2001;15:1288-1290.

39. Jun K, Piedras-Renteria ES, Smith SM, et al. Ablation of P/Qtype $\mathrm{Ca}(2+)$ channel currents, altered synaptic transmission, and progressive ataxia in mice lacking the alpha(1A)-subunit. Proc Natl Acad Sci U S A 1999;96:15245-15250.

40. Letts VA, Felix R, Biddlecome GH, et al. The mouse stargazer gene encodes a neuronal $\mathrm{Ca} 2+$-channel gamma subunit. Nat Genet 1998;19:340-347.

41. van den Maagdenberg AM, Pietrobon D, Pizzorusso T, et al. A Cacnala knockin migraine mouse model with increased susceptibility to cortical spreading depression. Neuron 2004;41:701710 .
42. Wakamori M, Yamazaki K, Matsunodaira H, et al. Single tottering mutations responsible for the neuropathic phenotype of the P-type calcium channel. J Biol Chem 1998;273:34857-34867.

43. Hans M, Luvisetto S, Williams ME, et al. Functional consequences of mutations in the human alpha1A calcium channel subunit linked to familial hemiplegic migraine. J Neurosci 1999; 19:1610-1619.

44. Wappl E, Koschak A, Poteser M, et al. Functional consequences of P/Q-type $\mathrm{Ca} 2+$ channel Cav2.1 missense mutations associated with episodic ataxia type 2 and progressive ataxia. J Biol Chem 2002;277:6960-6966.

45. Kraus RL, Sinnegger MJ, Glossmann H, Hering S, Striessnig J. Familial hemiplegic migraine mutations change alpha1A $\mathrm{Ca} 2+$ channel kinetics. J Biol Chem 1998;273:5586-5590.

46. Spacey SD, Hildebrand ME, Materek LA, Bird TD, Snutch TP. Functional implications of a novel EA2 mutation in the P/Q-type calcium channel. Ann Neurol 2004;56:213-220.

47. van den Maagdenberg AM, Kors EE, Brunt ER, et al. Episodic ataxia type 2 . Three novel truncating mutations and one novel missense mutation in the CACNA1A gene. J Neurol 2002;249: 1515-1519.

48. Walter JT, Alvina K, Womack MD, Chevez C, Khodakhah K. Decreases in the precision of Purkinje cell pacemaking cause cerebellar dysfunction and ataxia. Nat Neurosci 2006;9:389-397.

49. Weisz CJ, Raike RS, Soria-Jasso LE, Hess EJ. Potassium channel blockers inhibit the triggers of attacks in the calcium channel mouse mutant tottering. J Neurosci 2005;25:4141-4145.

50. Riess O, Schols L, Bottger H, et al. SCA6 is caused by moderate CAG expansion in the alpha1 A-voltage-dependent calcium channel gene. Hum Mol Genet 1997;6:1289-1293.

51. Kubodera T, Yokota T, Ohwada K, et al. Proteolytic cleavage and cellular toxicity of the human alpha1 A calcium channel in spinocerebellar ataxia type 6. Neurosci Lett 2003;341:74-78.

52. Matsuyama Z, Wakamori M, Mori Y, Kawakami H, Nakamura S, Imoto K. Direct alteration of the P/Q-type Ca2 + channel property by polyglutamine expansion in spinocerebellar ataxia 6 . J Neurosci 1999; 19:RC14.

53. Matsuyama Z, Yanagisawa NK, Aoki Y, et al. Polyglutamine repeats of spinocerebellar ataxia 6 impair the cell-death-preventing effect of $\mathrm{CaV} 2.1 \mathrm{Ca} 2+$ channel--loss-of-function cellular model of SCA6. Neurobiol Dis 2004;17:198-204.

54. Piedras-Renteria ES, Watase K, Harata N, et al. Increased expression of alpha $1 \mathrm{~A} \mathrm{Ca} 2+$ channel currents arising from expanded trinucleotide repeats in spinocerebellar ataxia type 6 . J Neurosci 2001;21:9185-9193.

55. Toru S, Murakoshi T, Ishikawa K, et al. Spinocerebellar ataxia type 6 mutation alters P-type calcium channel function. J Biol Chem 2000;275:10893-10898.

56. DiFiglia M, Sapp E, Chase KO, et al. Aggregation of huntingtin in neuronal intranuclear inclusions and dystrophic neurites in brain. Science 1997;277:1990-1993.

57. Verhoef LG, Lindsten K, Masucci MG, Dantuma NP. Aggregate formation inhibits proteasomal degradation of polyglutamine proteins. Hum Mol Genet 2002;11:2689-2700.

58. Bence NF, Sampat RM, Kopito RR. Impairment of the ubiquitinproteasome system by protein aggregation. Science 2001;292: $1552-1555$.

59. Goellner GM, Rechsteiner M. Are Huntington's and polyglutamine-based ataxias proteasome storage diseases? Int J Biochem Cell Biol 2003;35:562-571.

60. Chen H, Piedras-Renteria E. Altered frequency-dependent inactivation and steady state inactivation of polyglutamine-expanded alpha1A in SCA6. Am J Physiol Cell Physiol 2006.

61. Basu P, Chattopadhyay B, Gangopadhaya PK, et al. Analysis of CAG repeats in SCA1, SCA2, SCA3, SCA6, SCA7 and DRPLA loci in spinocerebellar ataxia patients and distribution of $\mathrm{CAG}$ repeats at the SCA1, SCA2 and SCA6 loci in nine ethnic populations of eastern India. Hum Genet 2000;106:597-604.

62. Gusella JF, Persichetti F, MacDonald ME. The genetic defect causing Huntington's disease: repeated in other contexts? Mol Med 1997;3:238-246.

63. Frontali M, Novelletto A, Annesi G, Jodice C. CAG repeat instability, cryptic sequence variation and pathogeneticity: evidence 
from different loci. Philos Trans R Soc Lond B Biol Sci 1999;354:1089-1094.

64. Kennedy L, Shelbourne PF. Dramatic mutation instability in HD mouse striatum: does polyglutamine load contribute to cellspecific vulnerability in Huntington's disease? Hum Mol Genet 2000;9:2539-2544.

65. Gatchel JR, Zoghbi HY. Diseases of unstable repeat expansion: mechanisms and common principles. Nat Rev Genet 2005;6:743755.

66. Berke SJ, Schmied FA, Brunt ER, Ellerby LM, Paulson HL. Caspase-mediated proteolysis of the polyglutamine disease protein ataxin-3. J Neurochem 2004;89:908-918.

67. Hackam AS, Singaraja R, Zhang T, Gan L, Hayden MR. In vitro evidence for both the nucleus and cytoplasm as subcellular sites of pathogenesis in Huntington's disease. Hum Mol Genet 1999; 8:25-33.

68. Kim YJ, Yi Y, Sapp E, et al. Caspase 3-cleaved N-terminal fragments of wild-type and mutant huntingtin are present in normal and Huntington's disease brains, associate with membranes, and undergo calpain-dependent proteolysis. Proc Natl Acad Sci U S A 2001;98:12784-12789.

69. Panet-Raymond V, Gottlieb B, Beitel LK, et al. Characterization of intracellular aggregates using fluorescently-tagged polyglutamine-expanded androgen receptor. Neurotox Res 2001;3:259275.

70. Becher MW, Kotzuk JA, Sharp AH, et al. Intranuclear neuronal inclusions in Huntington's disease and dentatorubral and pallidoluysian atrophy: correlation between the density of inclusions and IT15 CAG triplet repeat length. Neurobiol Dis 1998;4:387-397.

71. Cooper JK, Schilling G, Peters MF, et al. Truncated N-terminal fragments of huntingtin with expanded glutamine repeats form nuclear and cytoplasmic aggregates in cell culture. Hum Mol Genet 1998;7:783-790.

72. Davies SW, Turmaine M, Cozens BA, et al. Formation of neuronal intranuclear inclusions underlies the neurological dysfunction in mice transgenic for the HD mutation. Cell 1997;90:537548.

73. Emamian ES, Kaytor MD, Duvick LA, et al. Serine 776 of ataxin-1 is critical for polyglutamine-induced disease in SCA1 transgenic mice. Neuron 2003;38:375-387.

74. Fujigasaki H, Uchihara T, Koyano S, et al. Ataxin-3 is translocated into the nucleus for the formation of intranuclear inclusions in normal and Machado-Joseph disease brains. Exp Neurol 2000; $165: 248-256$.

75. Hackam AS, Hodgson JG, Singaraja R, et al. Evidence for both the nucleus and cytoplasm as subcellular sites of pathogenesis in Huntington's disease in cell culture and in transgenic mice expressing mutant huntingtin. Philos Trans R Soc Lond B Biol Sci 1999;354:1047-1055.

76. Huynh DP, Figueroa K, Hoang N, Pulst SM. Nuclear localization or inclusion body formation of ataxin-2 are not necessary for SCA2 pathogenesis in mouse or human. Nat Genet 2000;26:4450 .

77. Nucifora FC Jr, Ellerby LM, Wellington CL, et al. Nuclear localization of a non-caspase truncation product of atrophin-1, with an expanded polyglutamine repeat, increases cellular toxicity. J Biol Chem 2003;278:13047-13055.

78. Ordway JM, Tallaksen-Greene S, Gutekunst CA, et al. Ectopically expressed CAG repeats cause intranuclear inclusions and a progressive late onset neurological phenotype in the mouse. Cell 1997;91:753-763.

79. Perez MK, Paulson HL, Pendse SJ, Saionz SJ, Bonini NM, Pittman RN. Recruitment and the role of nuclear localization in polyglutamine-mediated aggregation. J Cell Biol 1998;143:14571470.

80. Peters MF, Nucifora FC, Jr, Kushi J, et al. Nuclear targeting of mutant Huntingtin increases toxicity. Mol Cell Neurosci 1999; $14: 121-128$.
81. Schmidt T, Landwehrmeyer GB, Schmitt I, et al. An isoform of ataxin-3 accumulates in the nucleus of neuronal cells in affected brain regions of SCA3 patients. Brain Pathol 1998;8:669-679.

82. Warrick JM, Paulson HL, Gray-Board GL, et al. Expanded polyglutamine protein forms nuclear inclusions and causes neural degeneration in Drosophila. Cell 1998;93:939-949.

83. Yang W, Dunlap JR, Andrews RB, Wetzel R. Aggregated polyglutamine peptides delivered to nuclei are toxic to mammalian cells. Hum Mol Genet 2002;11:2905-2917.

84. Nechiporuk T, Huynh DP, Figueroa K, Sahba S, Nechiporuk A, Pulst SM. The mouse SCA2 gene: cDNA sequence, alternative splicing and protein expression. Hum Mol Genet 1998;7:13011309.

85. Pulst SM, Santos N, Wang D, et al. Spinocerebellar ataxia type 2: polyQ repeat variation in the CACNA1A calcium channel modifies age of onset. Brain 2005;128:2297-2303.

86. De Jongh KS, Colvin AA, Wang KK, Catterall WA. Differential proteolysis of the full-length form of the L-type calcium channel alpha 1 subunit by calpain. J Neurochem 1994;63:1558-1564.

87. Hell JW, Westenbroek RE, Breeze LJ, Wang KK, Chavkin C, Catterall WA. N-methyl-D-aspartate receptor-induced proteolytic conversion of postsynaptic class C L-type calcium channels in hippocampal neurons. Proc Natl Acad Sci U S A 1996;93:33623367.

88. Scott VE, Felix R, Arikkath J, Campbell KP. Evidence for a 95 $\mathrm{kDa}$ short form of the alpha1A subunit associated with the omega-conotoxin MVIIC receptor of the P/Q-type Ca2+ channels. J Neurosci 1998;18:641-647.

89. Kordasiewicz HB, Thompson RM, Clark HB, Gomez CM. Ctermini of $\mathrm{P} / \mathrm{Q}$-type $\mathrm{Ca} 2+$ channel alpha1A subunits translocate to nuclei and promote polyglutamine-mediated toxicity. Hum Mol Genet 2006;15:1587-1599.

90. Hodel MR, Corbett AH, Hodel AE. Dissection of a nuclear localization signal. J Biol Chem 2001;276:1317-1325.

91. Jans DA, Xiao CY, Lam MH. Nuclear targeting signal recognition: a key control point in nuclear transport? Bioessays 2000;22: 532-544.

92. Moroianu J. Nuclear import and export pathways. J Cell Biochem 1999;32-33(suppl):76-83.

93. Gomez-Ospina N, Tsuruta F, Barreto-Chang O, Hu L, Dolmetsch $\mathrm{R}$. The $\mathrm{C}$ terminus of the L-type voltage-gated calcium channel ca(v)1.2 encodes a transcription factor. Cell 2006;127:591-606.

94. Klement IA, Skinner PJ, Kaytor MD, et al. Ataxin-1 nuclear localization and aggregation: role in polyglutamine-induced disease in SCA1 transgenic mice. Cell 1998;95:41-53.

95. Takeyama K, Ito S, Yamamoto A, et al. Androgen-dependent neurodegeneration by polyglutamine-expanded human androgen receptor in Drosophila. Neuron 2002;35:855-864.

96. Kohara K, Ono T, Tominaga-Yoshino K, Shimonaga T, Kawashima S, Ogura A. Activity-dependent survival and enhanced turnover of calcium in cultured rat cerebellar granule neurons. Brain Res 1998;809:231-237.

97. Toescu EC. Activity of voltage-operated calcium channels in rat cerebellar granule neurons and neuronal survival. Neuroscience 1999;94:561-570.

98. Mangiarini L, Sathasivam K, Seller M, et al. Exon 1 of the HD gene with an expanded CAG repeat is sufficient to cause a progressive neurological phenotype in transgenic mice. Cell 1996; 87:493-506.

99. Lin X, Antalffy B, Kang D, Orr HT, Zoghbi HY. Polyglutamine expansion down-regulates specific neuronal genes before pathologic changes in SCA1. Nat Neurosci 2000;3:157-163; Comment Nat Neurosci 2000;3:103-4.

100. Masliah E. Recent advances in the understanding of the role of synaptic proteins in Alzheimer's disease and other neurodegenerative disorders. J Alzheimers Dis 2001;3:121-129.

101. Selkoe DJ. Alzheimer's disease is a synaptic failure. Science 2002;298:789-791. 